\title{
An Edge-Fog Computing Framework for Cloud of Things in Vehicle to Grid Environment
}

\author{
Neeraj Kumar, Tanya Dhand, Anish Jindal, Gagangeet Singh Aujla, Haotong Cao, Longxiang Yang*
}

\begin{abstract}
The penetration of electric vehicles (EVs) embedded with information and communication technology (ICT) devices and tools form a huge connected network that can be viewed as Internet-of-EVs (IoEV). The huge data gathered in IoEV network needs to be processed at cloud-based infrastructure which has abundant resources. However, due to the high mobility of the EVs, resource management from the remote cloud service providers has become one of the most difficult tasks to be performed in this environment. In this regard, data analytics fused with fog or edge computing can be leveraged to increase the resource availability in V2G environment where resources are provided to the EVs on the edge of the network. Keeping these points in mind, this paper presents a new framework for integration of cloud computing and IoEV on the edge of the network which provides flexibility to the end users for smooth execution of various applications. In addition, a resource allocation and job scheduling strategy for EVs at the edge of the network is presented in the paper. The results obtained with respect to various performance metrics confirm the applicability of the proposed scheme for future applications in V2G scenario.

Index Terms-Cloud of things, fog computing, Internet-of-
\end{abstract} electric vehicles, resource management, vehicle to grid.

\section{INTRODUCTION}

$\mathbf{F}$ ROM the last few decades, there has been an exponential growth in the usage of smart devices for the purpose of communication and information sharing. This revolution where various types of smart devices communicate with each other irrespective of their geographical locations is known as Internet to Things (IoT). With the evolution of Internet technology in recent years, each object/device in IoT environment is capable of performing computing and communication; one such object is an electric vehicle (EV) [1, 2]. EVs are environment friendly vehicles and can be perceived as an intelligent objects as they are capable of performing computing and communication tasks. For example, EVs can receive and send the information related to the energy to a centralized controller.

In vehicle to grid (V2G) environment, vehicles provide bidirectional communication between the grid and the end users for the exchange of energy. The vehicles participate in

N. Kumar and T. Dhand are with the Computer Science \& Engineering Department, Thapar Institute of Engineering \& Technology, Patiala, India. (e-mail: neeraj.kumar@thapar.edu, tanyadhand75@gmail.com)

A. Jindal is with the School of Computer Science and Electronic Engineering, University of Essex, Colchester, UK. (e-mail: anishjinda190@ gmail.com)

G. S. Aujla is with the School of Computing, Newcastle University, Newcastle upon Tye, UK. (e-mail: gagi_aujla82@yahoo.com)

H. Cao and L. Yang are with the College of Telecommunications \& Information Engineering, Nanjing Universiy of Posts and Telecommunications, China. (e-mail: caohaotong@163.com, yanglx@njupt.edu.cn)

*Corresponding author energy transfer from service providers (i..e., grid) and service consumers (i.e., the end users). However, to make a decision about when and where this energy is to be transferred is a challenging task. The large-scale presence of EVs connected to the grid via communication technology in the V2G network can be perceived as the Internet of EVs (IoEV) network. There are various constraints in this IoEV network with respect to the energy transfer between grid and EVs. Firstly, EVs have high mobility which causes a frequent network disconnection. Secondly, these vehicles have less storage space to accommodate a large data in their database. Thirdly, EVs have less computing power for handling large database query. Lastly, the integration and interoperability of EVs to the grid for energy transfer is another issue. So, to handle these issues, a unified framework is required in which there exists a smooth and efficient communication between EVs and grid [3, 4, 5, 6, 7].

Hence, to provide resources to the EVs for performing the tasks of energy transfer and job scheduling, computing and communication facilities need to be on the edge of the network so as to reduce the delay during access to these resources $[8,9]$. This type of computing paradigm is called as fog computing which has emerged as one of the most powerful technologies of the modern computing era. In fog computing, all the resources are available at the edge of the networks to facilitate the end users for smooth execution of services. The integration of smart devices with cloud infrastructure using Internet is called as cloud of things (CoT). In CoT, devices connected to centralized cloud infrastructure using Internet are allowed to share their information in a unified manner. These devices may be located anywhere in the network from where these can access various services provided by the cloud infrastructure. However, if all the resources are available at the edge of the network, then the delay to access all such resources would be minimal which ultimately improves any implemented solution in this environment. Fig. 1 shows a generalized framework in which there exists an interaction between IoT, smart devices, and fog/edge environment.

There are three major components in the fog-based infrastructure for providing services to the end users. These components are broker, resource pool, and resource scheduler. The broker acts as an facilitator for the interaction between the gateway network and the resource pool (which is allocated to end devices by the resource scheduler). Fig. 1 shows the interaction between edge gateways, smart devices, and fog infrastructure occurs at various levels. Nevertheless, there are various challenges and constraints with respect to the implementation of any designed solution in the V2G environment in 


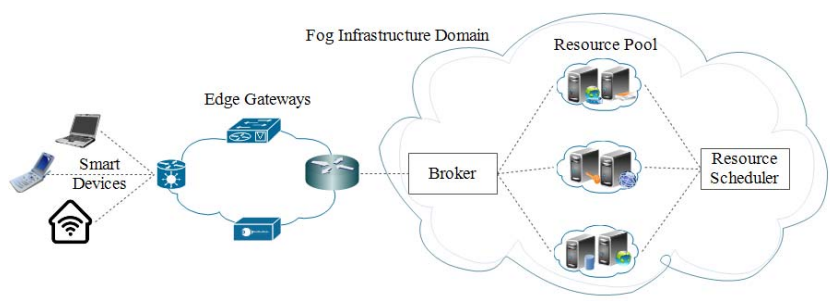

Fig. 1: Interaction between CoT and fog computing.

the fog computing era. The vehicles are having high mobility, so the data transfer from EVs to edge router needs to be done taking into consideration of this aspect. The pricing policy from the utilities which are connected to the nano data centers varies with respect to the duration and number of EVs entering or leaving the network. The type and format of the data vary with respect to the vehicles used for testing any implemented solution in this environment.

To tackle these challenges, a scheme is proposed in this paper for efficient resource management at the edge of the network which provides efficient communication services to EVs in IoEV and V2G environments. The major contributions of the proposed scheme are summarized as follows.

1) A novel architecture for connecting the moving objects like EVs is presented in the fog computing environment.

2) Using this architecture is used for energy trading in IoEV network using fog computing is elaborated where $\mathrm{EVs}$ act as the mobile chargers for energy transfer to charging stations.

3) With respect to the mobility of the EVs, the resource allocation and scheduling policy is designed with respect to the constrained optimization problem in the fog computing environment.

Rest of the paper is organized as follows. Section 2 describes the system model. Section 3 discusses the resource allocation and job scheduling at the edge as a constrained convex optimization problem. Section 4 shows the obtained results. Finally, Section 5 concludes the article.

\section{System MOdEL FOR IOEV NETWORK}

Figure 1 shows the general architecture used for the interaction of CoT with fog computing. At the more granular level, the detailed system model used in the proposed scheme for the IoEV network is illustrated in Fig. 2.

\section{A. Components}

Figure 2 consists of various components of the proposed system model used for interaction between different entities in V2G environment. As shown in this figure, the model is presented as a hierarchical architecture in which EVs are located at the lowest level (which are sources of data generation and consumption). These EVs participate to seek various services such as energy trading between vehicles and the grid by forming different coalitions. The coalitions among the EVs are formed based upon the payoff utility function. These coalitions may vary from hundreds to thousands in each city depending upon the size of the city which would generate huge data at a very fast pace. This data collected from the EVs in various coalitions is sent to the fog gateways which are located at different geographical areas for processing and analysis. These fog gateways are the entry points of information received from the EVs. From different edge domains at the edge gateways, the information is passed onto the traffic controller which monitors the data and performs data analytics to take energy trading decisions at the edge layer. It also includes the resource allocation and VM scheduling procedures (details of which are given in next section) for smooth execution of various applications. Finally, the information is sent to the centralized controller which is the overall manager of all types of resource management and data storage at the top level $[10,11,12]$.

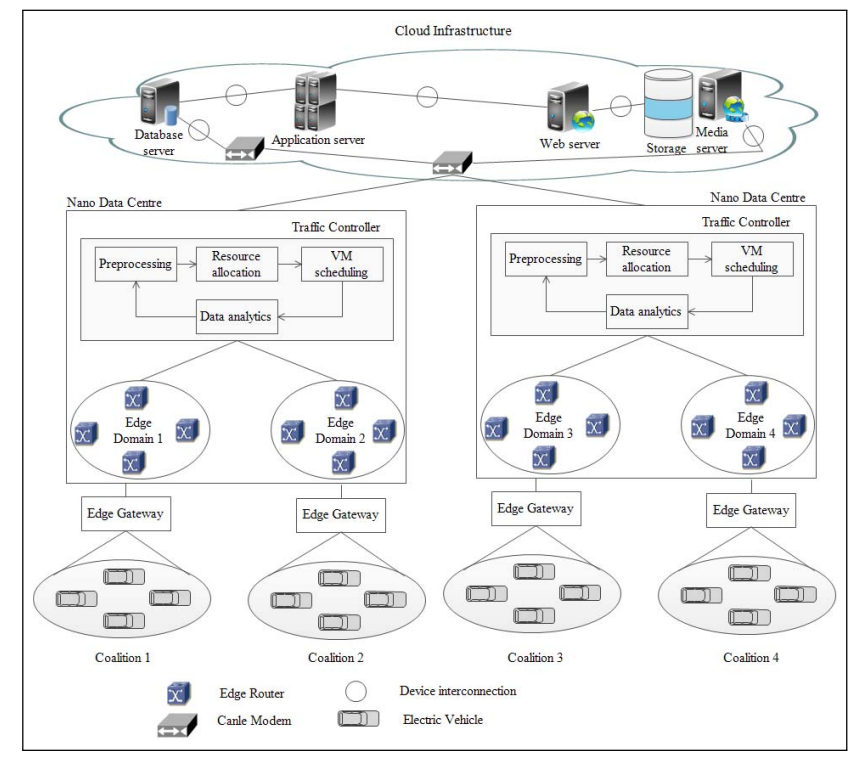

Fig. 2: System model for cloud of things and fog computing.

\section{B. Providing service}

The major aim of this fog computing architecture is to use it for providing various services in the IoEV network. In this regard, an application of this paradigm is discussed in this section which focuses on energy trading in $\mathrm{V} 2 \mathrm{G}$ environment. The application scenario of $\mathrm{V} 2 \mathrm{G}$ environment is considered to comprise of various EVs which can give the energy back to the grid through the charging stations that are available in the nearby regions. As there are thousands of EVs present in a typical city, the data is gathered from them at a rapid pace which needs to be processed at an equal pace in order to maintain the load profile of the grid. The delay in processing the data at the cloud may result in miscommunication of the available power which can further result in grid imbalance. Therefore, the data processing should happen at an expeditious rate so as to take quick decision regarding the energy trading of EVs with the grid. To facilitate this quick decision making, 
most of the communication devices are located on the edge of the network in fog computing for running various applications in $\mathrm{V} 2 \mathrm{G}$ environment.

For energy trading, EVs act as the mobile chargers which provide (or takes) energy to (or from) the charging station located in the city. These charging stations are directly connected with the grid which can pump back the energy to the grid if required. Whenever the grid has excess energy or requires energy to fulfill the load demand, the EVs are utilized to flatten the overall load profile of the grid. For this purpose, a cooperative coalition game among various EVs has been formulated. In the proposed coalition game, each EVs is assumed to be player in the game having a utility value based upon the remaining energy. Initially, a coalition among the EVs is formed using the payoff function defined by the remaining energy of the EV and the distance it can travel. This payoff utility function is mathematically defined as follows.

$$
P F_{i}^{\psi}=n^{\phi} \times \frac{D}{E_{i}^{r e m}}
$$

where, $n^{\phi}$ is the number of attempts made by the EV with the cloud to seek services, $D$ and $E_{i}^{r e m}$ are the distance of EV from the charging station and remaining energy in the EVs, respectively. Based on these factors, decisions about the energy transfer are made in the proposal such that the utility of coalition increases after taking the energy trading decisions.

\section{Data analytics at the Edge}

In the proposed solution, EVs have been considered as the energy carriers for distribution of energy to the charging stations and the data from the EVs is collected and processed on the devices located at the edge of the network. For this purpose, the following two cases are considered in which the requests of the EVs are processed at the edge of the network.

Case 1 - Peak hours: In the peak hours, there is a sudden increase in the number of requests for energy usages from different energy sectors. So, a balance is required to be maintained at the grid with respect to generation and consumption. To depict the same, following equation is used.

$$
\begin{aligned}
& \operatorname{Cap}(t)=\underbrace{\left(E_{i}^{\text {Win }(t)}+E_{i}^{P V(t)}\right)}_{\text {gen }}-\underbrace{\left(E_{i}^{\text {cons }}\right)}_{\text {cons }} \\
& \text { s.t. et } t_{i}= \begin{cases}1, & \| \text { gen }, \text { cons } \| \leq P F_{i}^{\psi} \\
0, & \| \text { gen }, \text { cons } \|>P F_{i}^{\psi}\end{cases}
\end{aligned}
$$

where, $\operatorname{Cap}(t)$ is the total energy capacity available at the grid, $E_{i}^{W i n(t)}$ and $E_{i}^{P V(t)}$ depict the energy generation sources of wind and PV panels respectively, and $E_{i}^{\text {cons }}$ represents the overall load demand consumption at the grid. The difference between consumption and generation is modeled for computing the value of energy transfer signal, $e t_{i}$, from the overall energy capacity available at any instant $t$. Based on this difference, the value of $e t_{i}$ is decided (it is the binary variable whose value 1 or 0 ) which is used to decide the direction of flow of energy for each EV, i.e., from EV to the grid or from grid to the EV. This calculation is also dependent upon the individual $P F_{i}^{\psi}$ of the EV so that based upon its value, each EV decide its action of energy transfer. Eq. (2) is used to make adaptive decisions with respect to energy management in the peak hours when there is a huge demand for the energy from different sources.

Case 2 - Off-peak hours: During the off-peak hours, the demand for the energy is less and generation may be high. In such cases, extra energy may be stored at the battery energy storage system $(B E S S)$ present at the charging stations (which can be utilized when there is an increase in the demand for the energy). The EVs are also utilized in this case as the latency associated with the energy transfer from the charging station to EVs is less. These EVs may be charged from BESS located at different charging stations or can be directly charged from the grid supply as per the load demand on the grid. The following equation is used to depict this scenario.

$$
e t_{i}= \begin{cases}B E S S, & \| \text { gen }, \text { cons } \|<0 \\ E v_{i}, & \| \text { gen }, \text { cons } \| \geq 0\end{cases}
$$

This equation shows both the scenarios of energy transfer of either to BESS or from EVs depending upon the difference between demand and supply at the grid. Based upon these two scenarios, intelligent decisions about the energy transfer are made in the proposal. For example, if there is an excess energy from generation units, then it can be used to charge $E v_{i}$, otherwise, the stored energy in the $B E S S$ can be utilized.

To take the energy trading decisions, all the data communication requests from the EVs are processed at the edge of the network. At the edge, the resources are allocated to all the participating EVs in a manner that a large number of requests can be handled in less time duration. In this way, the communication delay would be minimum and the EVs can be directed more quickly to the nearby charging stations. This, in turn, helps the grid to recover quickly from the load instability which is a very crucial aspect in V2G environment. For the communication purposes, edge resources are allocated to the EVs using an adaptive scheduling strategy and the workload at the edge is managed as given in the next section.

\section{RESOURCE ALLOCATION AND SCHEDULING AT THE EDGE}

The service-oriented resource manager and scheduler are designed to provide better services to IoEV nodes/things located at the edge of the network. The resources can be of any type, i.e., storage resources, I/O resources, computation resource, and other network resources. Figure 3 describes the generalized framework used for service-oriented resource manager and scheduler. The working of various components of this framework is explained as follows.

Before any of the user's requests are sent for resource allocation, the parameters such as available bandwidth, number of virtual machines (VMs), and type of VMs are registered in dynamic resource allocation index service (RIS). After accounting for all the present resources, user's request is forward to the resource pool. With every resource pool, there is a fog message exchanger associated with it, which is 


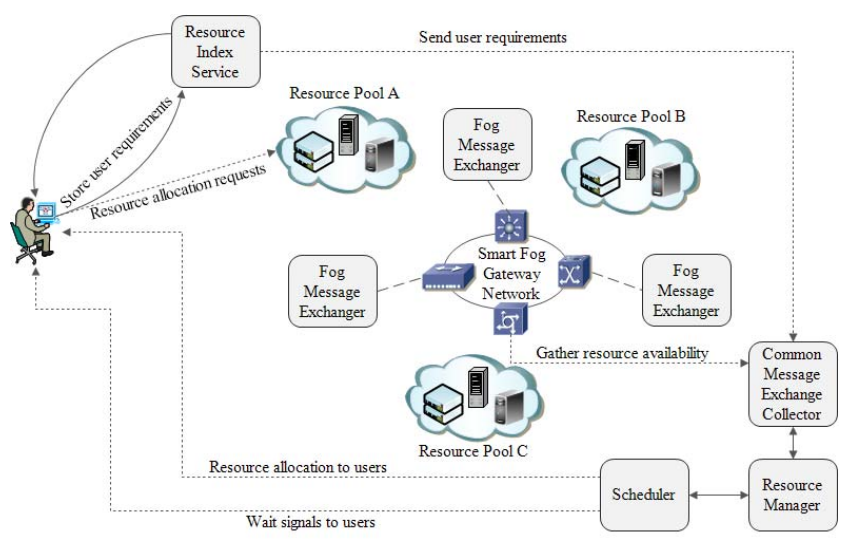

Fig. 3: Resource allocation and scheduling framework.

interconnected with resource pool to collect the number of free and available resources, i.e., the resources which are underutilized. Fog message exchanger identifies the unused VMs on the fog resource pool. It is important to monitor the resource usage so that the unused or underused resources can be scheduled in an effective and efficient way. This, in turn, would increase the quality of service by decreasing the overall delay and increasing the network throughput.

The fog message exchangers of different resource pools are interconnected via smart gateways. These gateways help them to communicate with one another from a network domain to another network domain, so that the fog message exchanger discovers the total number of free and available resources distributed across various resource pools. The free and available resource list is then passed onto the common message exchange collector (CMEC). Along with that, the CMEC also receives the user's requirements through network link from RIS, where the user's requirements get registered. The CMEC aims to find a best match of freely available resources based on the information stored in RIS and creates the group of free resources which are likely to provide best service. At last, if the resources are available, a successful signal is passed to the users to indicate that the resources are available which can be used by different users to execute their applications. Otherwise, the user request is queued up and a wait signal is sent to the user if the number of free resources does not satisfy user's requirements.

\section{A. Workflow management at the Edge}

Workflow management is the sequence of activities performed for successful execution of various services in the network. Although it is easy to construct a workflow in the dedicated cloud computing based infrastructure, however, with the high mobility of the EVs, it is a challenging task to execute a workflow at the edge of the network. The following sequence of activities is performed in the proposed solution for managing the workflow at the edge of the network as shown in Fig. 4.

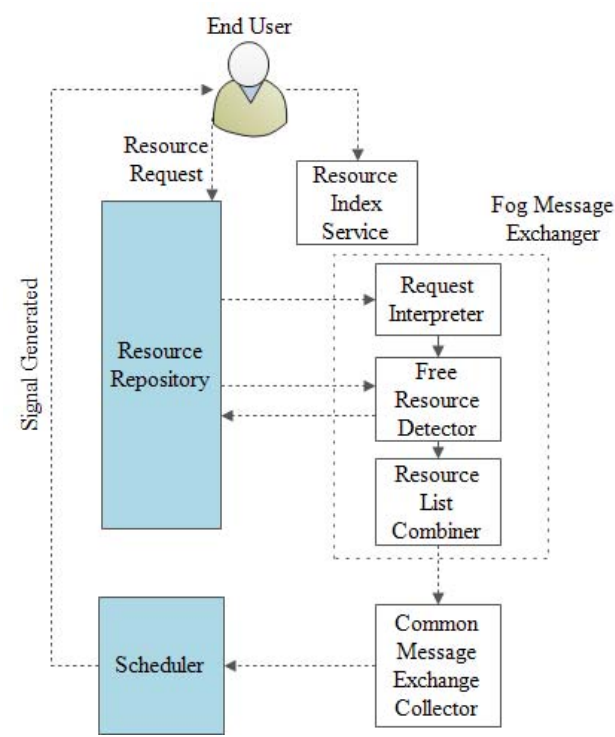

Fig. 4: Workflow management.

(i) User Request Classification: Initially, the users' requests are classified into two levels. At the first level, we have considered a scenario when no data resources are requested by the users. At the other level, a scenario has been considered where the end users are demanding a finite number of resources. In both of these cases, resource requests are identified by RIS.

(ii) Requirement Identification: This step is followed for the users classified at the second level. In this step, the user requests for $N$ number of resources; these requests are stored in the RIS. RIS registers the user resource request on the basis of the quality of service metrics such asnumber of requests, current load, and type of service needed.

(iii) Count Resource Availability: All $N$ resource requests are then passed to resource pool repository which contains the information about the used and unused resources. This repository is attached with a fog message exchanger which further contains request interpreter, free resource detector, and resource list combiner as shown in Fig. 4. The request interpreter converts the users' requirements into the quality of service metrics such as number of VMs required, time delay deadline, bandwidth requirement, cost of transmission, and latency. The free resource detector identifies the resources needed by the user and makes a list of all such resources. This list contains all freely available resources. The resource list combiner merges all the available resources which satisfy the user requirements as per the metrics specified above, which are then forwarded to CMEC.

(iv) Resource Allocation: In this stage, the scheduler allocates the resources to the user for task completion. The two approaches can be used for the purpose of resource selection and scheduling in the scheduler which are discussed as 
follows.

(a) Random Resource Selection - In this strategy, resources are randomly chosen from the set of available resources for allocation without considering the quality of service metrics.

(b) Dynamic Resource Selection - This selection is based on quality of service metrics which select resources intelligently based on the utility of the different players as defined in the Eq. (1).

In the proposed scheme, resource allocation at the edge is performed using the dynamic resource allocation policy, which takes the quality of service metrics into consideration.

\section{RESULTS AND DISCUSSIONS}

The results obtained using the proposed scheme for allocating resources at the edges by utilizing fog computing environment in IoEV network are discussed in this section.

\section{A. Simulation settings}

We have considered the application scenario as shown in Fig. 2 for conducting the simulation experiments. For this purpose, $2000 \mathrm{EVs}$ are used which have different battery capacities and a cloud server connected to 10 nano data centers at the edge of the network. For simulating the fog computing environment, $50 \mathrm{VMs}$ of 2GB capacities are also considered in this proposal. The total energy capacity available in the IoEV network is computed using Eq. (2). The proposed scheme is evaluated by using mac NS-2. The performance metrics considered in this work are:

- Delay incurred: It is the time taken by the EVs for bidirectional energy transfer requests at the edge of the network.

- Throughput: It is the total amount of packets delivered and received from the edge devices at any instant.

- Overhead generated: It is defined as the extra number of packets sent to the edge of the network at the expense of main packets.

- Execution time: The total time taken to execute a particular service in the fog computing environment.

\section{B. Performance evaluation}

1) Impact on delay incurred: Figure 5a shows the delay incurred with respect to the energy trading requests from the EVs to the edge of the network. This figure clearly indicates that there is less delay incurred in comparison to the case when the proposed scheme is not applied to process the requests at the nano data centers placed at the edge of the network. The reason for such behavior is due to the quick decision making in the proposed scheme with respect to the payoff utility of each player. The decisions with respect to energy trade are taken keeping in view of the load on the edge network.
2) Impact on throughput generated: Figure 5b shows the impact of the proposed scheme on the throughput generated with respect the execution of a finite number of requests generated from the edge devices and EVs present in different coalitions as shown in Fig. 2. The results obtained indicate that by using the proposed scheme, there is an increase in the throughput generated at the edge with respect to the energy demand satisfaction from the vehicles. This proves the effectiveness of the proposed scheme as compared to the case where the proposed scheme is not used. The main reason behind this phenomenon is the adaptive resource management and scheduling in the proposed scheme for various jobs/requests generated from the EVs. Hence, there is an increase in the throughput generated with respect to the requests generated for energy trading from the EVs.

3) Impact on overhead generated: Figure 6a shows the overhead generated with respect to the energy transfer requests from the EVs to the edge of the network. The overhead generated is counted with respect to the extra messages transferred to the cloud nano centers at the edge of the network. To handle all such requests, an efficient resource allocation and scheduling strategy is used in this proposal. Moreover, a workflow management based upon the payoff utility of the players (EVs) is utilized. These factors have a direct impact on the faster execution of various jobs at the edge of the network which results in a less overhead generation. Hence, the proposed scheme generates considerably less overhead as compared to the case when the proposed scheme is not considered.

4) Impact on execution time: Figure $6 \mathrm{~b}$ shows the impact of the execution time with the increase in the number of VMs in the fog computing environment. The results obtained clearly indicates the superior performance of the proposed scheme as compared to the traditional network. It is mainly due to an increase in more number of available resources at the edge of the network due to which the performance of the proposed scheme is better than the existing scheme.

\section{CONCLUSION}

In fog computing, most of the resources for successful execution of various applications are available at the edge of the network. In this paper, we have analyzed fog computing and its applicability in $\mathrm{V} 2 \mathrm{G}$ environment. For this purpose, a unified framework for integration of fog computing in $\mathrm{V} 2 \mathrm{G}$ environment is presented. The applicability of the proposed scheme has been tested in the V2G scenario. These decisions are based on the coalition game theory in which EVs are assumed to have a payoff function. This payoff function is then used for taking intelligent decisions with respect to the bidirectional energy flow between EVs and grid during peak and off-peak hours. Moreover, a cloud resource management and scheduling scheme is also presented to allocate various fog resources to the participating entities. To achieve this task, a workflow schedule management process at the edge is also defined in this paper. A case study for evaluation is also presented to test the effectiveness of the proposed scheme 


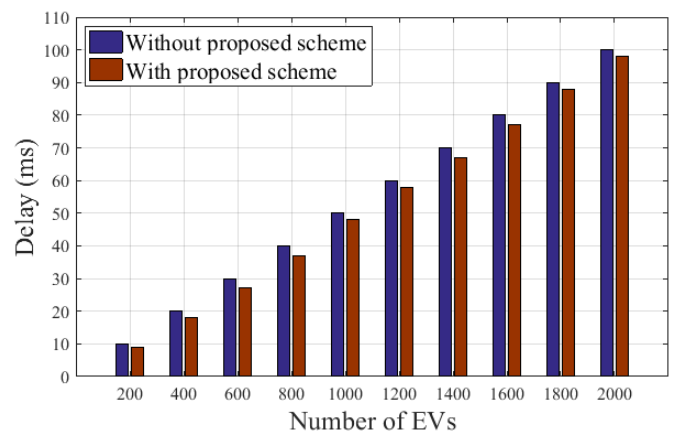

(a)

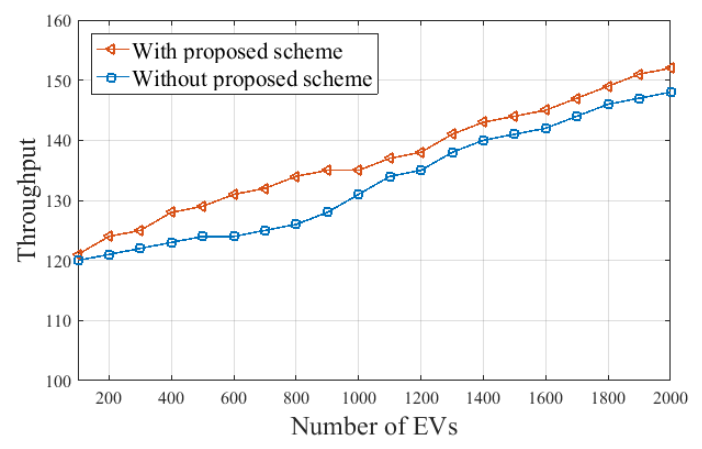

(b)

Fig. 5: Analysis of (a) Delay incurred with number of PHEVs. (b) Throughput generated with number of PHEVs.

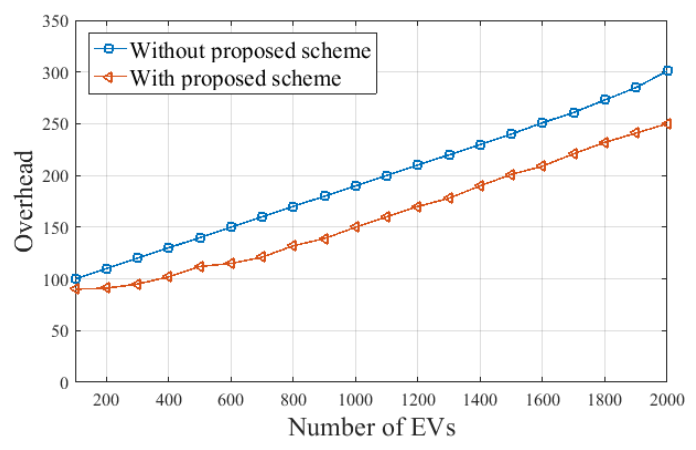

(a)

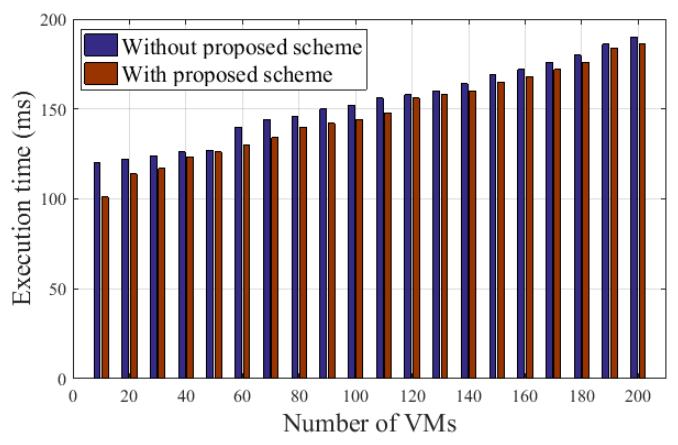

(b)

Fig. 6: Analysis of (a) Overhead generation with number of PHEVs. (b) Execution time with number of PHEVs.

using fog computing architecture. The evaluation results indicate the effectiveness of the proposed scheme with respect to performance metrics such as-delay incurred, throughput, overhead generated, and execution time.

In future, the security features can be explored with respect to the proposed scheme by integrating them with advanced communication technologies such as $5 \mathrm{G}$.

\section{REFERENCES}

[1] D. Dong, F. Luo, X. Zhang, D. Boroyevich, and P. Mattavelli, "Grid-interface bidirectional converter for residential dc distribution systems-part 2: Ac and dc interface design with passive components minimization," IEEE Transactions on Power Electronics, vol. 28, no. 4, pp. 1667-1679, 2013.

[2] N. Kumar, S. Misra, J. Rodrigues, J.-H. Lee, M. S. Obaidat, and N. Chilamkurti, "Playing the smart grid game: performance analysis of intelligent energy harvesting and traffic flow forecasting for plug-in electric vehicles," IEEE Vehicular Technology Magazine, vol. 10, no. 4, pp. 81-92, 2015.

[3] H. Liu and B. He, "F2c: enabling fair and fine-grained resource sharing in multi-tenant iaas clouds," IEEE Transactions on Parallel and Distributed Systems, vol. 27, no. 9, pp. 2589-2602, 2016.

[4] G. S. Aujla, A. Jindal, and N. Kumar, "Evaas: Electric vehicleas-a-service for energy trading in sdn-enabled smart transportation system," Computer Networks, vol. 143, pp. 247-262, 2018.
[5] X. Dai, J. M. Wang, and B. Bensaou, "Energy-efficient virtual machines scheduling in multi-tenant data centers," IEEE Transactions on Cloud Computing, vol. 4, no. 2, pp. 210-221, 2016.

[6] R. Yu, G. Xue, V. T. Kilari, and X. Zhang, "Network function virtualization in the multi-tenant cloud," IEEE Network, vol. 29, no. 3, pp. 42-47, 2015.

[7] N. Kumar, S. Zeadally, N. Chilamkurti, and A. Vinel, "Performance analysis of bayesian coalition game-based energy-aware virtual machine migration in vehicular mobile cloud," IEEE Network, vol. 29, no. 2, pp. 62-69, 2015.

[8] T. X. Tran, A. Hajisami, P. Pandey, and D. Pompili, "Collaborative mobile edge computing in $5 \mathrm{~g}$ networks: New paradigms, scenarios, and challenges," IEEE Communications Magazine, vol. 55, no. 4, pp. 54-61, 2017.

[9] A. Jindal, G. S. Aujla, and N. Kumar, "Survivor: A blockchain based edge-as-a-service framework for secure energy trading in sdn-enabled vehicle-to-grid environment," Computer Networks, vol. 153, pp. 36-48, 2019.

[10] G. S. Aujla, N. Kumar, A. Y. Zomaya, and R. Ranjan, "Optimal decision making for big data processing at edge-cloud environment: An sdn perspective," IEEE Transactions on Industrial Informatics, vol. 14, no. 2, pp. 778-789, 2017.

[11] A. Gelman, J. B. Carlin, H. S. Stern, D. B. Dunson, A. Vehtari, and D. B. Rubin, Bayesian data analysis. CRC press Boca Raton, FL, 2014, vol. 2.

[12] W. Xu, W. Liang, X. Lin, and G. Mao, "Efficient scheduling of multiple mobile chargers for wireless sensor networks," IEEE Transactions on Vehicular Technology, vol. 65, no. 9, pp. 76707683, 2016. 